\title{
Delta-like Factor 1 as a Possible Therapeutic Target for Sarcomas
}

\author{
Han-Soo Kim, MD ${ }^{*}{ }^{\dagger}$, Sun Hee Ahn, $\mathrm{MS}^{*}$, Ha Jeong Kim, PhD*, \\ Jong Woong Park, $\mathrm{MD}^{*}$, Ilkyu Han, $\mathrm{MD}^{*, \dagger}$ \\ ${ }^{*}$ Department of Orthopedic Surgery, Seoul National University Hospital, Seoul, \\ ${ }^{\dagger}$ Department of Orthopedic Surgery, Seoul National University College of Medicine, Seoul, Korea
}

Background: Cancer stem cells (CSCs) are cells characterized by their self-renewal and tumorigenic potential. The purpose of this study was to discover the role of the delta-like factor 1 (DLK1) in sarcoma.

Methods: mRNA expression of DLK1 from 13 sarcoma cell lines was examined. Isolated CSCs from the tumors were examined using fluorescence-activated cell sorting (FACS) with CD133, the CSC marker, or sphere-forming assay. The relationship between DLK1 and CSCs in sarcoma was examined using cell proliferation and cell invasion assays after they were treated with DLK1 short interfering RNA (siRNA).

Results: A high expression of DLK1 mRNA was observed in all sarcoma cell lines. However, CSCs were isolated from over expressed sarcomas of the DLK1 gene, and they have shown to be expressed lower than the wild type. The anti-cancer effects of DLK1 siRNA inhibited cell proliferation and invasion in U20S, A204, and sw872. In addition, treatment with DLK1 siRNA inhibited cell invasion in sw872 CSCs. DLK1 gene induces tumorigenesis in various sarcoma cells and regulates the invasiveness of liposarcoma. These results suggest that DLK1 could serve as a possible therapeutic target for sarcoma.

Conclusions: Our study showed that the DLK1 gene induces tumorigenesis in various sarcomas and is associated with invasive mechanism in sarcoma. These results suggest DLK1 could serve as a possible therapeutic target in a variety of sarcomas.

Keywords: Sarcoma, DLK1, CD133, Cancer stem cell, Tumorigenesis

Sarcomas represent a group of cancers that exhibit mesenchymal differentiation, accounting for approximately $1 \%$ of all adult malignancies. ${ }^{1)}$ Despite aggressive surgical resection with adjuvant chemotherapy and radiotherapy, about $30 \%$ of patients with sarcoma still experience local relapse or metastasis. In general, prognoses of patients with metastatic sarcoma remain poor, with the 3-year survival rate of $20 \%-30 \% .{ }^{2)}$ Therefore, novel approaches to treatment are needed to improve the outcomes of these patients.

Received July 23, 2019; Accepted February 6, 2020

Correspondence to: Ilkyu Han, MD

Department of Orthopedic Surgery, Seoul National University Hospital, 101 Daehak-ro, Jongno-gu, Seoul 03080, Korea

Tel: +82-2-2072-0682, Fax: +82-2-764-2718

E-mail: hik19@snu.ac.kr
Cancer stem cells (CSCs) constitute the minority of cells within the tumor, but they have unique characteristics such as the self-renewal and tumorigenic potential. Due to these characteristics, the established anti-cancer drugs are unable to remove the CSCs completely and thus are unable to protect against cancer reoccurrence. Therefore, in order to remove the CSCs completely, it is necessary to have a comprehensive understanding of the signaling pathways involved in cancer cell maintenance or regulation.

Recently, studies on CSCs, which focused on finding molecular biological characteristics or cell signaling pathways that maintain and regulate $\mathrm{CSCs},{ }^{3)}$ have found that the self-renewal signal of CSCs is modified in various cancer species. The study of the Notch and Sonic Hedgehog signaling pathway is 1 such study. ${ }^{4}$ Since first confirmed in acute myeloid leukemia, CSCs have been detected in breast, brain, and colon cancer and have been identified in 
Kim et al. Delta-like Factor 1 as a Possible Therapeutic Target

Clinics in Orthopedic Surgery • Vol. 12, No. 3, $2020 \bullet$ www.ecios.org

a variety of solid tumors. Sarcoma CSCs have been found in osteosarcoma, synovial sarcoma, and undifferentiated pleomorphic sarcoma. ${ }^{5-7)}$ Therefore, the study of CSCs is of major interest in cancer therapy.

Delta-like factor 1 (DLK1), also known as preadipocyte factor 1 (pref-1), was cloned in the preadipocyte cells 3T3-L1 cDNA library. Previous studies showed the role of DLK1 in mesenchymal stem cell differentiation, adipogenesis, myogenesis, and osteoblastogenesis. ${ }^{8,9)}$ In addition, it has been reported that the overexpression of DLK1 is associated with the development of various cancers including blood cancer, breast cancer, colon cancer, pancreatic cancer, and glioma. ${ }^{10)}$ DLK1 has been shown to be associated with the maintenance of CSCs. For example, in liver cancer, cells that expressed DLK1 had CSC characteristics. Suppression of DLK1 expression resulted in depression of liver cancer tumorigenesis due to the decreased number of CSCs. ${ }^{11)}$ In addition, suppressing DLK1 induced the differentiation of undifferentiated cancer cells in neuroblastoma, glioma, and blood cancer. ${ }^{12)}$ However, little is known about the role of DLK1 in sarcoma progression. With this regard, we investigated the expression of DLK1 and the role of DLK1 in the maintenance of sarcomas and sarcoma CSCs.

\section{METHODS}

\section{Cell Culture}

Cell lines were purchased from ATCC Cell Bank, Lonza Clonetics (HS-SY-II) and the culture media used were Dulbecco's Modified Eagle Medium (DMEM; Gibco, Grand Island, NY, USA), Macoy5A (Gibco; A204, Saos2, SK-ES1), and L15 (sw872, sw1353, sw684, sw982), supplemented with $10 \%$ fetal bovine serum (FBS), $2 \mathrm{mM}$ glutamine, $100 \mathrm{U} / \mathrm{mL}$ penicillin, and $100 \mu \mathrm{g} / \mathrm{mL}$ streptomycin. Cells were cultured at $37^{\circ} \mathrm{C}$ in a $5 \% \mathrm{CO}_{2}$ incubator and the medium was changed twice a week.

\section{RNA Extraction and Quantitative Real-Time Polymerase Chain Reaction}

Total RNA was extracted from 14 sarcoma, osteoblast, and fibroblast cell lines using RNeasy Mini Kit (Qiagen, Hilden, Germany) according to the manufacturer's instructions. Complementary DNA was synthesized using a SuperScript VILO cDNA synthesis kit (Invitrogen, Waltham, MA, USA). Quantitative real-time polymerase chain reaction (PCR) was performed using the Taqman ABI 7500 (Applied Biosystems, Lincoln, CA, USA) with a probe mixture. The primers and probes were designed using Primer Express Software 2.0 (Applied Biosystems).
Fragments of the DLK1 sequence were amplified using a forward primer (5'-GCC ACA GCA CCT ATG GTG AG-3'), a reverse primer (5'-TAA GAC GGC AAA GTC CC-3'), and a probe (5'-FAM-GTGTGATCAACGGCTCCCCCTGCCA-TAMRA-3'). The probe sequence, 5'-AGC ACA GCT CCG TCG CCG AGT G-3', was labeled at the $5^{\prime}$ end with a reporter fluorophore and on the 3 ' end with a quencher fluorophore TAMRA. Glyceraldehyde-3-phosphate dehydrogenase (GAPDH) was used as an internal standard. Quantitative amplification reactions were performed as follows: 2 minutes at $50^{\circ} \mathrm{C}, 10$ minutes at $95^{\circ} \mathrm{C}$, and 4515 -second cycles at $95^{\circ} \mathrm{C}$, and 1 minutes at $60^{\circ} \mathrm{C}$. The quantitative analysis of mRNA was performed using the ABI Prism 7500 Software ver. 2.0.5 (Applied Biosystems). To determine the relative quantification of mRNA expression, the standard curve method was used. GAPDH was run concurrently as a housekeeping gene.

\section{Western Blot Analysis}

For Western blot analyses, cells were lysed in RIPA buffer (Sigma-Aldrich, Laramie, WY, USA) with complete mini protease inhibitors (Roche, Indianapolis, IN, USA). The proteins were quantified using the Bradford assay (BioRad, Hercules, CA, USA), separated using NU-PAGE gel (NOVEX) electrophoresis and transferred to nitrocellulose membranes (Amersham Hybond; GE Healthcare, Chicago, IL, USA). Following blocking with 5\% skimmed milk in TBS (0.1\% Tween 20), membranes were left overnight at $4^{\circ} \mathrm{C}$ in an anti-DLK1 primary antibody solution (1:100; Millipore, Burlington, MA, USA). The anti- $\beta$-actin antibody (Santa Cruz Biotechnology, Santa Cruz, CA, USA) was used as a housekeeping molecule. These blots were then labeled with horseradish peroxidase-conjugated secondary antibodies (Santa Cruz Biotechnology) for 1 hour at $4^{\circ} \mathrm{C}$. The membranes were washed with TBST (Tris Buffered Saline with Tween 20, 0.1\%; Sigma-Aldrich) and the target protein was detected by an enhanced chemiluminescence Western blot detection system (GE, Berlin, Germany). The relative expression level of each protein was calculated by comparing $\beta$-actin expression level.

\section{Cancer Stem-Like Cells Sorting in Sarcoma Cell Lines}

Cells were detached from culture plates using $0.02 \%$ ethylenediaminetetraacetic acid (EDTA) in phosphate-buffered saline (PBS) and washed twice in PBS containing 0.1\% bovine serum albumin (BSA). At least $5 \times 10^{5}$ cells were incubated in $100 \mu \mathrm{L}$ of $0.1 \%$ BSA/PBS buffer with monoclonal antibodies in a 1:10 dilution or isotype antibody for 20 minutes at $4^{\circ} \mathrm{C}$ in the dark. The antibodies used were mouse anti-human CD133/2-PE conjugated and isotype- 
Kim et al. Delta-like Factor 1 as a Possible Therapeutic Target

Clinics in Orthopedic Surgery • Vol. 12, No. 3, $2020 \bullet$ www.ecios.org

PE conjugated as a control (Miltenyi Biotec, Bologna, Italy). After washing, the labeled cells were analyzed by flow cytometry using a fluorescence-activated cell sorting (FACS) cell sorter (FACS ARIA II; BD Bioscience, San Jose, CA, USA). CD133+ cells were sorted for experiments and CD133- cells were harvested as control. Seven days after sorting, cells were detached and stocked. The isolated cells were cultured for 1 week under the same conditions as the cell line. Experiments using the CD133+ cells were conducted between passages 4 and 6 to avoid their differentiation into the cancer cell.

\section{Sphere-Forming Assay}

Cells were seeded at a density of 60,000 cells/well in 6-well ultra-low attachment plates (Corning Life Science, Tewksbury, MA, USA) containing $3 \mathrm{~mL}$ DMEM/F12 supplemented with $1 \%$ methylcellulose, progesterone $(10 \mathrm{nM})$, putrescine $(50 \mu \mathrm{M})$, sodium selenite $(15 \mathrm{nM})$, transferrin $(13 \mu \mathrm{g} / \mathrm{mL})$, insulin $(10 \mu \mathrm{g} / \mathrm{mL})$, human epidermal growth factor (EGF; $10 \mathrm{ng} / \mathrm{mL}),(10 \mathrm{ng} / \mathrm{mL}$ ), and human bFGF (10 ng/mL; Sigma-Aldrich) and cultured at $37^{\circ} \mathrm{C}$ in a $5 \%$ $\mathrm{CO}_{2}$ incubator for 72 hours. Fresh aliquots of EGF and bFGF were added every other day. Spheres were visible by inverted microscopes.

For the sphere-forming assay, cells were cultured for a further 2 to 3 days. Sphere cells were collected with a 40$\mu \mathrm{m}$ cell strainer, which is used to remove single cells, and then counted under a microscope. ${ }^{2)}$ Spheres were separated into single cells by pipetting and then stored in liquid nitrogen $\left(\mathrm{LN}_{2}\right)$ to maintain the viability of the cancer stemlike cells (CSLCs) for other experiments. Experiments using the spheres were carried out within cell passage 6 .

\section{Soft-Tissue Assay}

To determine the difference in tumorigenic potential between the 2 cell fractions, CD133+ cells, wild-type cells, and sphere-forming cells were seeded in soft agar at a density of 1,000 cells/well in 24-well plates in triplicate. For the base layer, $2.4 \%$ agar stock solution was melted in a microwave oven, cooled to $40^{\circ} \mathrm{C}$ in a water bath and then mixed with culture medium to obtain a solution of $0.8 \%$ agar in DMEM. This solution $(0.5 \mathrm{~mL} /$ well $)$ was then added to 24-well plates. For the top layer, the agar stock solution was diluted with culture medium to obtain a solution of $0.3 \%$ agar in DMEM. This solution $(0.5 \mathrm{~mL} /$ well $)$ with the cells was gently mixed and an aliquot was added into the 24-well plates. Cells were plated and incubated for 21 days at $37^{\circ} \mathrm{C}$ in a humidified atmosphere of $5 \% \mathrm{CO}_{2}$ and 50 $\mu \mathrm{L}$ DMEM. At the end of the incubation period, colonies were stained with $0.005 \%$ crystal violet solution and large colonies were counted using an inverted microscope. ${ }^{13)}$

\section{Transfection with DLK1 Short Interfering RNA}

The DLK1 gene was silenced using short interfering RNA (siRNA). The dsRNA human DLK1 mRNA sequence and complementary dsRNA sequence were as follows: 5'-CUGGCCGUCAACAUCAUCUdTdT-3', 5'-AGAUGAUGUU GACGGCCAGdTdT-3', 5'-GUGAACGCUACGCUUACA UdTdT-3', 5'-AUGUAAGCGUAGCGUUCACdTdT-3' and 5'-CUCUCUCUUAAUGCAUGAUdTdT-3', 5'-AUCAUGCAUUAAGAGAGdTdT-3'.

The negative control siRNA duplex (scramble RNA) used was 5'-GCG CGC UUU GUA GGA UUC GdTdT-3' and 5'-CGA AUC CUA CAA AGC GCG CdTdT-3'. U2OS, sw872, and A204 cells were plated at a density of $1 \times 10^{5}$ cells in a medium containing 10\% FBS in 6-well culture plates for 24 hours. The cells were washed with PBS the next day, treated for 20 minutes with a mixture of DLK1 siRNA or negative control siRNA and lipofectamine 2000 (Invitrogen) in Opti-MEM (Gibco) medium to induce cell transfer, and were cultured for a further 48 hours at $37^{\circ} \mathrm{C}$ in a humidified atmosphere of $5 \% \mathrm{CO}_{2}$ after adding a normal medium. Inhibition of the gene was confirmed by real-time PCR (data not shown). The proliferation and invasion assays were studied to elucidate the functional role of DLK1.

\section{Cell Proliferation Assay}

Cells were seeded onto 96-well plates at a density of 2,500 cells/well. DLK1 was suppressed with siRNA and then the cells were incubated at $37^{\circ} \mathrm{C}$ in a $5 \% \mathrm{CO}_{2}$ incubator for 72 hours. In order to observe cell proliferation, $10 \mu \mathrm{L}$ of WST1 reagent solution was added to each well (Roche) and then the culture was incubated in the dark at $37^{\circ} \mathrm{C}$ in a $5 \%$ $\mathrm{CO}_{2}$ incubator for 4 hours. The absorbance was measured at $440 \mathrm{~nm}$ by a microplate reader (Molecular Devices, San Jose, CA, USA).

\section{Invasion Assay}

The in vitro invasive properties of sarcomas and CSLCs from sarcoma cell lines were evaluated using the transwell invasion assay (Becton-Dickinson, Bedford, MA, USA). PET membranes ( $8 \mu \mathrm{m}$ pore size) of the upper compartment of 24-well transwell culture chambers were coated with Matrgel (BioCoat Matrigel Invasion Chambers; Corning Life Science, Tewksbury, MA, USA). Cells $(1 \times$ $10^{5}$ ) suspended in $300 \mu \mathrm{L}$ of serum-free medium were applied to the upper compartment and the lower compartment was filled with $750 \mu \mathrm{L}$ of DMEM with $10 \%$ FBS, which acted as a chemoattractant. The chamber was incu- 
Kim et al. Delta-like Factor 1 as a Possible Therapeutic Target

Clinics in Orthopedic Surgery • Vol. 12, No. 3, $2020 \bullet$ www.ecios.org

bated for 16 to 18 hours at $37^{\circ} \mathrm{C}$ to allow cells to migrate to the lower chamber. After incubation, the non-invading cells were removed from the upper surface of the filter by scrubbing. The number of invading cells, which were stained with diff-Quick stain kit (Dade Behring Inc., Newark, DE, USA), was counted using a microscope.
A

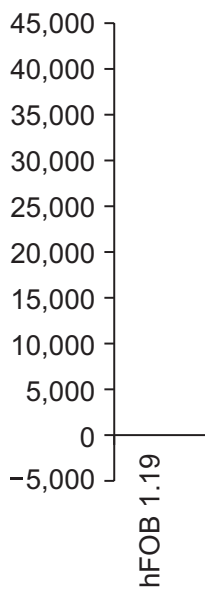

B

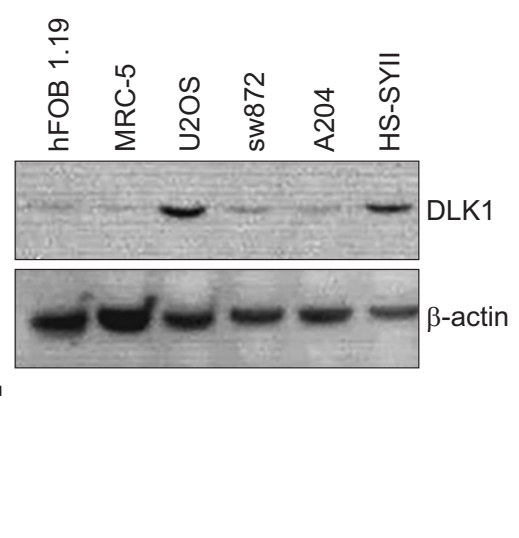

Fig. 1. Delta-like factor 1 (DLK1) expression level in sarcoma cell lines. Human osteoblast cell line (hFOB 1.19) was used as the control cell line. (A) Quantification of mRNA using real-time polymerase chain reaction $\left(^{*} p<0.05\right)$. (B) Protein analysis by Western blotting.

\section{A}
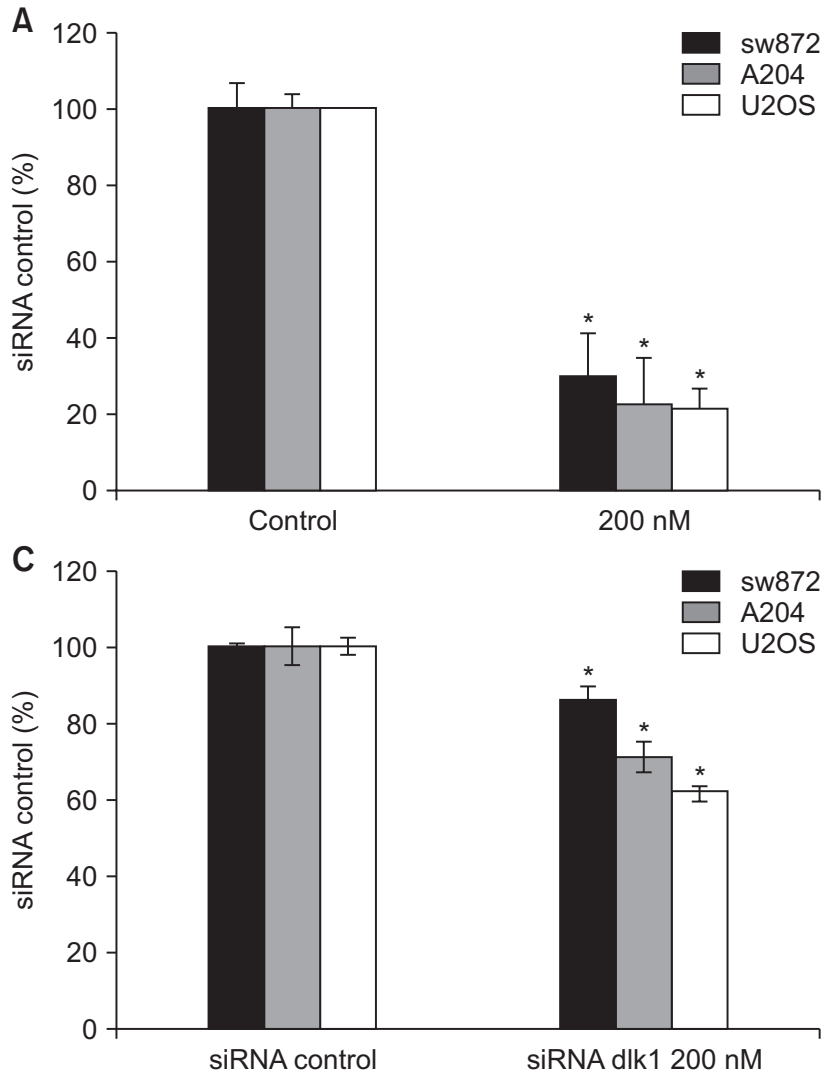

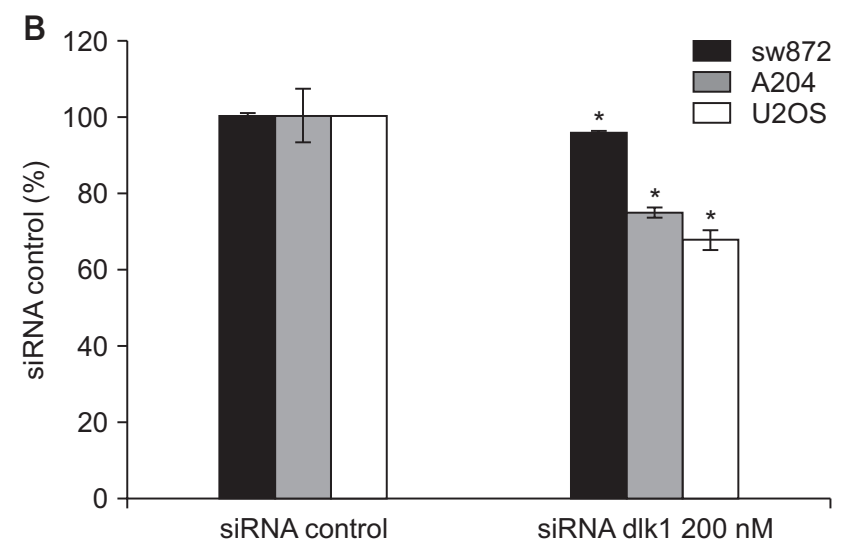

Fig. 2. Delta-like factor 1 (DLK1) gene effect comparison after treatment with DLK1 siRNA (200 nM) in sarcoma cell lines. (A) Detection of DLK1 gene inhibition using real-time polymerase chain reaction $(p<0.05)$. (B) The result from the cell proliferation assay $(p<0.05)$. (C) The result from the cell invasion assay $(p<0.05)$. Error bars show standard deviation. siRNA: short interfering RNA. *The average contact pressure was the highest for the $80^{\circ}$ flat liner, followed in descending order by the difference was statistically significant with $p<0.05$. 
Kim et al. Delta-like Factor 1 as a Possible Therapeutic Target

Clinics in Orthopedic Surgery • Vol. 12, No. 3, $2020 \bullet$ www.ecios.org

\section{Statistical Analysis}

Paired $t$-tests, calculated using IBM SPSS ver. 21.0 (IBM Corp., Armonk, NY, USA), were used to evaluate the difference in response between samples and control. Data are presented as mean and standard deviation and are representative of multiple independent experiments. A $p$-value of $<0.05$ was considered statistically significant.

\section{RESULTS}

\section{DLK1 Expression Levels in Sarcoma Cell Lines}

Real-time PCR confirmed the increased expression of DLK1 mRNA in sarcoma cell lines when compared to the normal bone cell line (1.19 hFOB). In particular, DLK1 mRNA was overexpressed in U2OS (osteosarcoma), sw872 (liposarcoma), A204 (rhabdomyosarcoma), and HS-SYII (synovial sarcoma) cells. However, no significant difference in DLK1 mRNA expression was found between the different sarcoma subtypes. Western blot analyses were performed using sarcoma cell lines that expressed high levels of DLK1 mRNA. We found that levels of protein expression were lower than those of mRNA expression in sw872 and A204 cells. There was no correlation between the level of protein and mRNA expressed in sarcoma cell lines (Fig. 1).

\section{Comparison of DLK1 Gene Effects in Sarcoma Cell Lines}

DLK1 was suppressed in cells using a DLK1 siRNA. We observed a reduction in cell mRNA level, proliferation, and cell invasion in U2OS, A204, and sw872. Although DLK1 suppression reduced both proliferation and invasion, the invasive ability of cells was more strongly reduced. Interestingly, cell proliferation was only slightly decreased in sw872 after DLK1 suppression (Fig. 2).

\section{CSC Isolation in Sarcoma Cell Lines}

CSCs were selected from U2OS, A204, and sw872 by the FACS sorting method using the CD133 cell surface molecule, which is known as a CSC marker in sarcoma. ${ }^{14)}$ For further experiments, CSCs were used within 6 passages to avoid their differentiation into cancer cells. Sarcospheres were isolated by sphere-forming assay using CD133-/+ cells generated by FACS sorting. Active sarcopheres, spheres that were $40 \mu \mathrm{m}$ or more in size, were collected (Fig. 3).

We investigated CSC characteristics-self-renewal
A

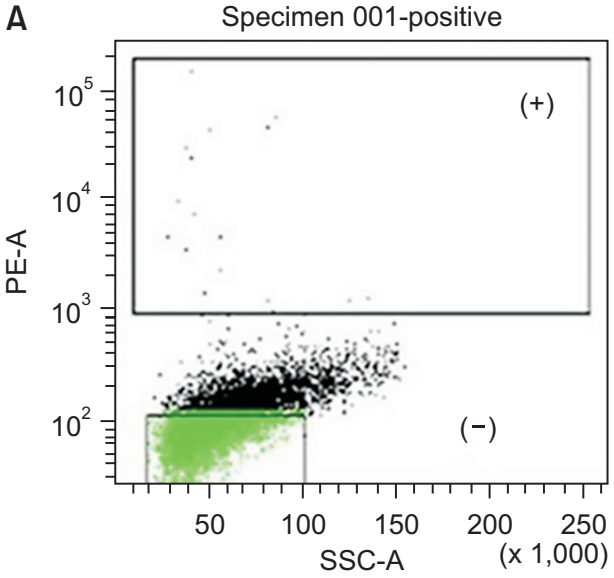

B CD133- cell
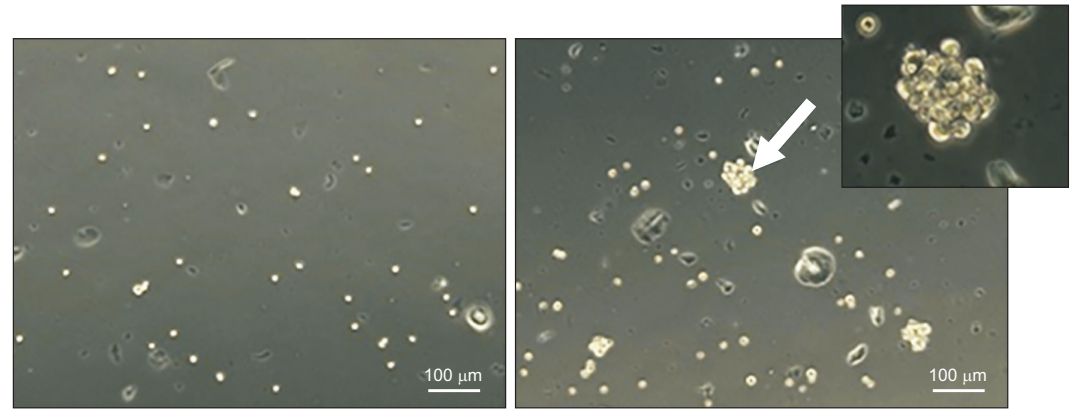

C
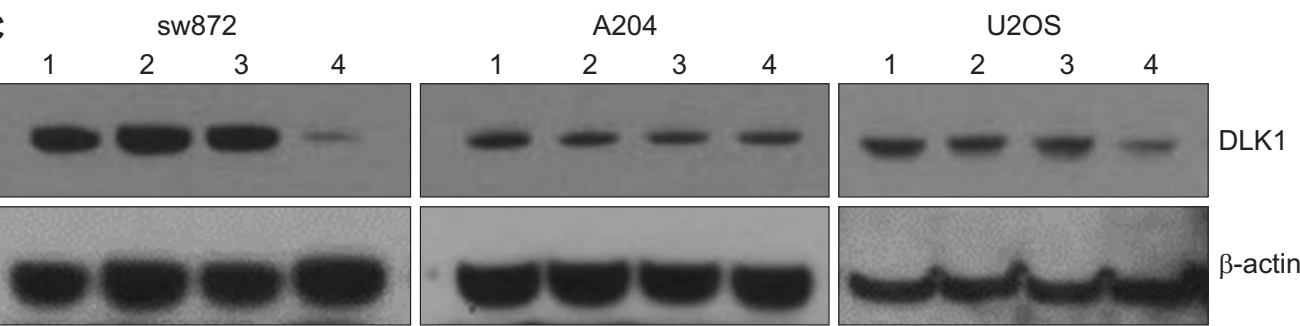

Fig. 3. Differences in delta-like factor 1 (DLK1) expression between wild-type cells, CD133- cells, and cancer stem cells (CSCs) (CD133+, sarcospheres). (A) CD133+/- region for fluorescence-activated cell sorting in sw872 cells. (B) Microscopic image of sphere-forming cells incubated on a low attached plate for 3 days. (C) A comparison of protein levels using Western blot. Group 1: wild type, group 2: CD133- cell, group 3: CD133+ cell (CSCs), group 4: spheres (CSCs). Scale bar, $100 \mu \mathrm{m}$. 
Kim et al. Delta-like Factor 1 as a Possible Therapeutic Target

Clinics in Orthopedic Surgery • Vol. 12, No. 3, $2020 \bullet$ www.ecios.org
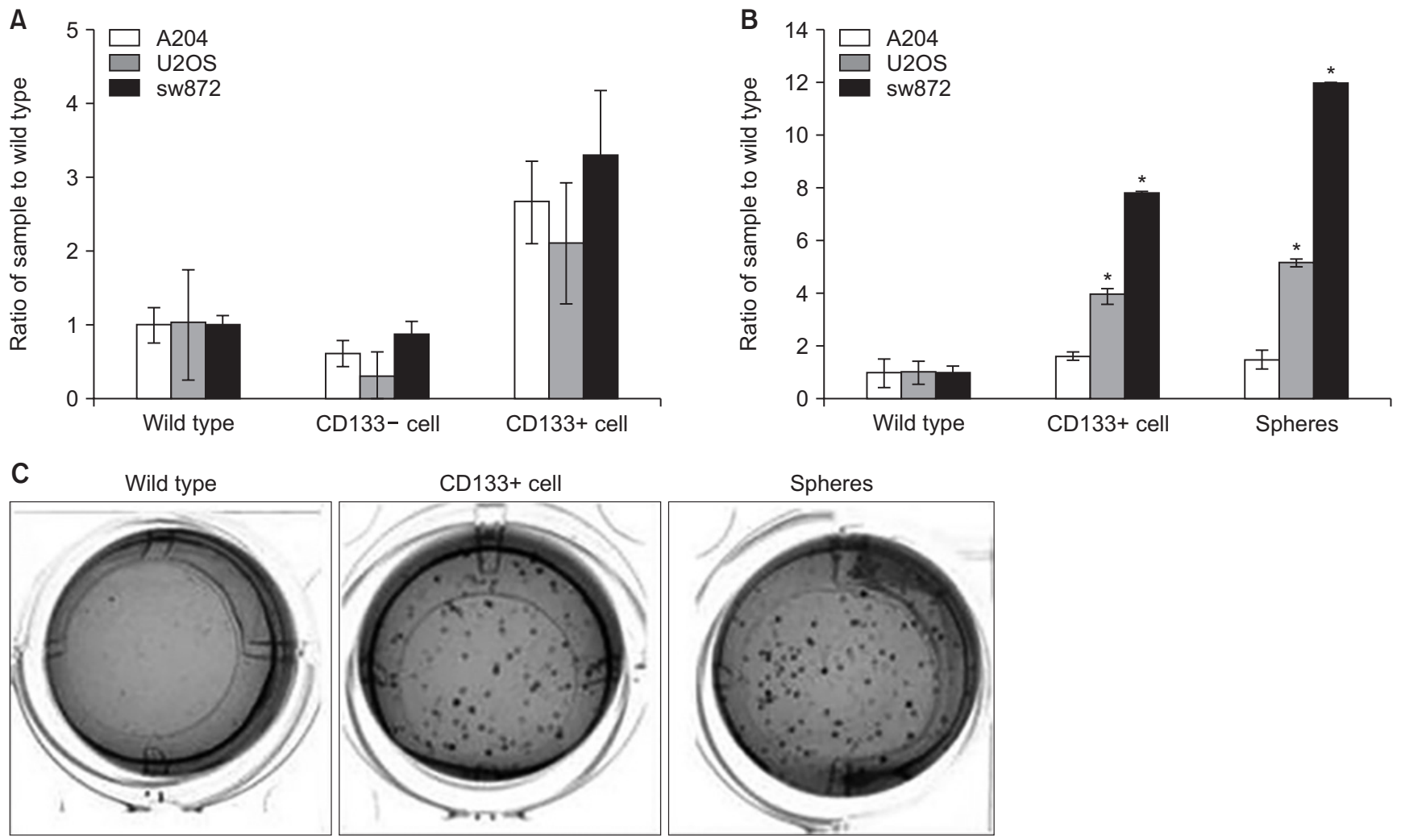

Fig. 4. Isolation of cancer stem cells in sarcoma cell lines. (A) The number of spheres formed after the cells were incubated on a low attached plate for 5 days $(p<0.05)$. (B) The number of colonies formed after the cells were incubated in soft agar for 3 weeks $\left(^{*} p<0.05\right)$. (C) A photo of the stained colonies in 6-well plates after the soft agar assays.
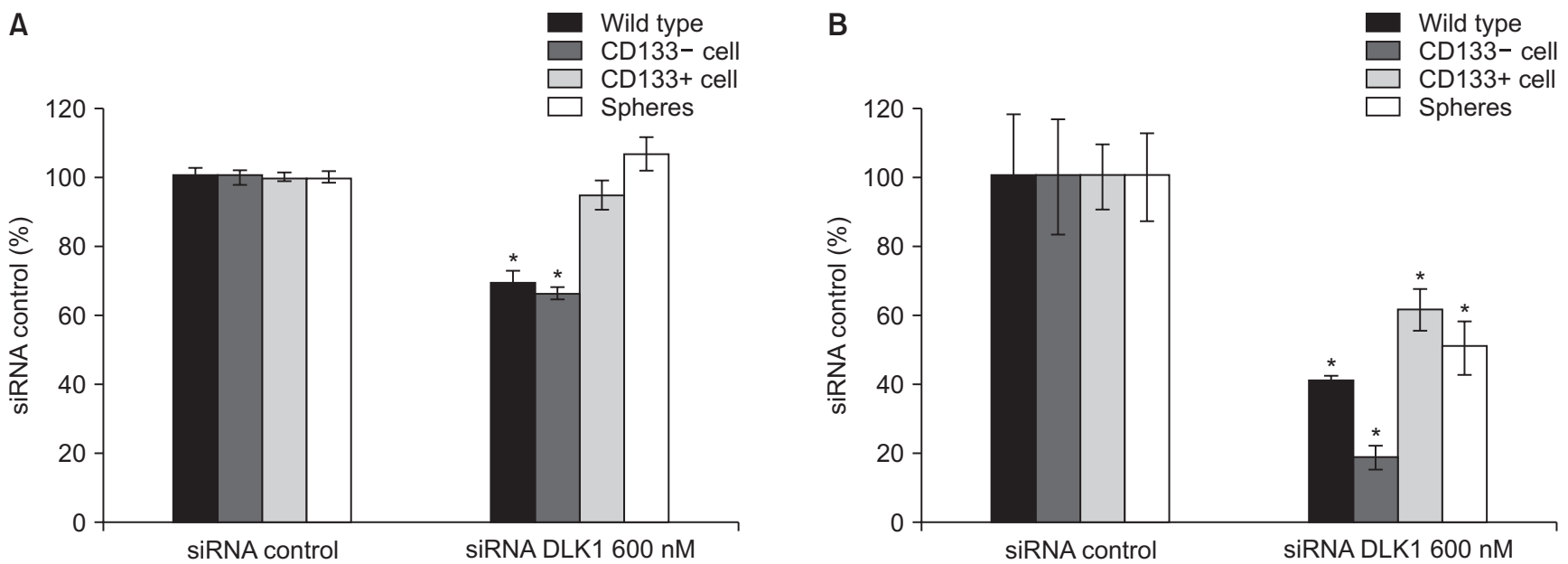

Fig. 5. Relationship between delta-like factor 1 (DLK1) and cancer stem cells in sw872 (liposarcoma). (A) The result of the cell proliferation assay ( ${ }^{*} p<$ 0.05). (B) The result of the cell invasion assay $\left({ }^{*} p<0.05\right)$. siRNA: short interfering RNA.

and tumorigenesis-by sphere-forming and soft agar assays. A comparative test between CD133-/+ and wildtype cells revealed that $\mathrm{CD} 133+$ cells formed many more spheres than CD133- and wild-type cells during the sphere-forming assay. Soft agar assays, which were con- ducted to observe the tumorigenic potential of the CSCs, revealed the presence of many more colonies in CD133+ cells than in CD133- and wild-type cells. These results show that CD133+ cells exhibit strong CSC characteristics. However, we found that CD133+ and CD133- A204 cells 
Kim et al. Delta-like Factor 1 as a Possible Therapeutic Target

Clinics in Orthopedic Surgery • Vol. 12, No. 3, $2020 \bullet$ www.ecios.org

both formed a large number of colonies (Fig. 4).

\section{Relationship between DLK1 Gene Effect and sw872 (Liposarcoma) CSCs}

To investigate the relationship between DLK1 and CSCs in sw872 cells where the DLK1 mRNA was highly expressed, we divided sw872 cells into 4 groups; wild type, CD133-, CD133+, and spheres. Western blot was used to detect the DLK1 gene in all 4 groups. We observed that the DLK1 expression level in sw872 CSCs was relatively low (Fig. 3).

Cells in all 4 groups were then treated with high doses of DLK1 siRNA (600 nM) to suppress DLK1 expres- sion and observe any changes in cell proliferation and invasion. We found that suppression of DLK1 inhibited the invasiveness of CSLCs more than their ability to proliferate. In particular, the suppression of DLK1 or siRNA DLK1 inhibited more effectively activation of invasive cells in sw872 (liposarcoma) without CSCs (Figs. 5B and 6) as well as cell proliferation (Fig. 5A).

\section{DISCUSSION}

Despite the local control obtained by surgery and radiation therapy, up to $40 \%$ of soft-tissue sarcoma patients eventu-

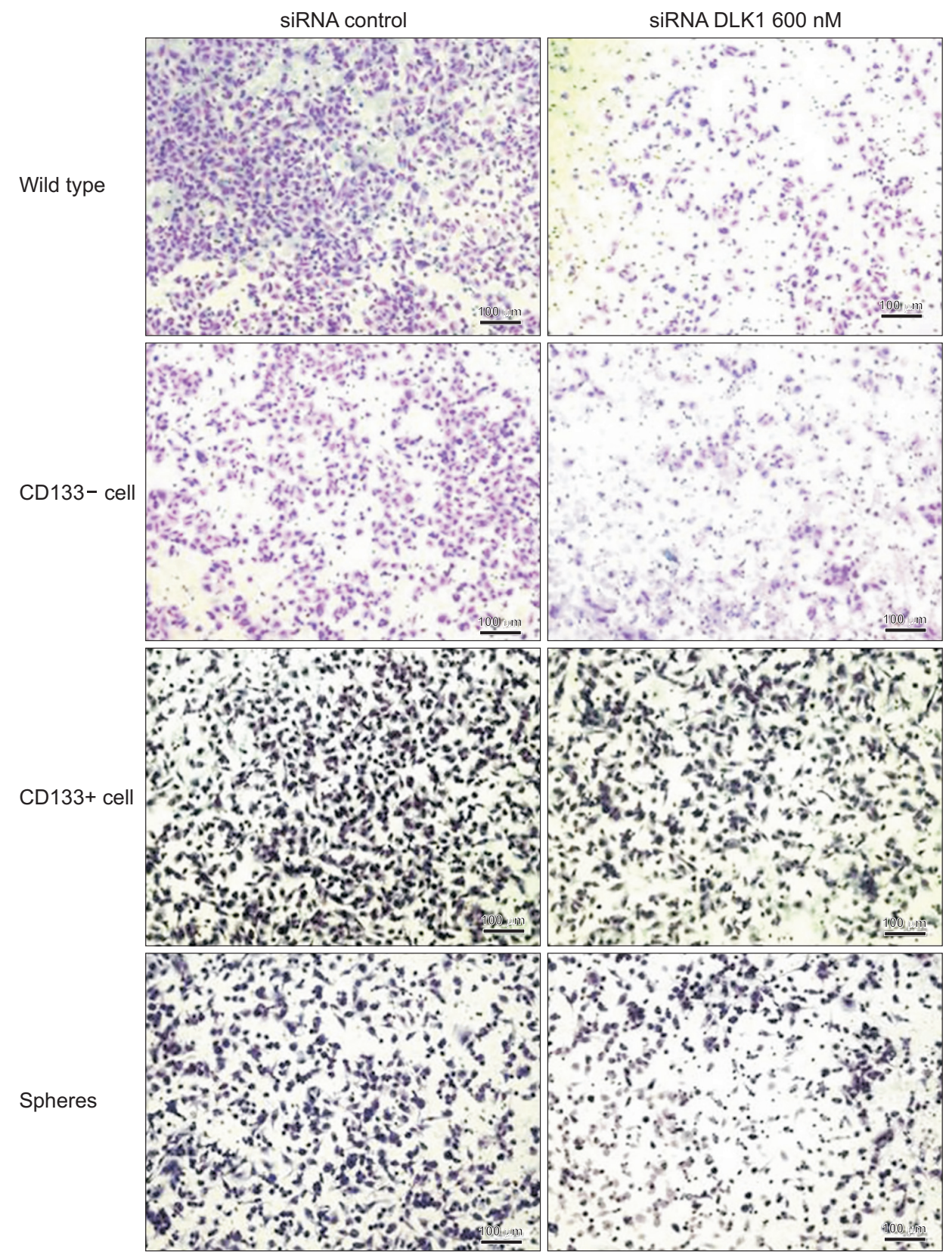

Fig. 6. The microscopic image of the cell invasion assay used to investigate the relationship between delta-like factor 1 (DLK1) and cancer stem cells in sw872 (x10). Scale bar, $100 \mu \mathrm{m}$. siRNA: short interfering RNA. 
Kim et al. Delta-like Factor 1 as a Possible Therapeutic Target

Clinics in Orthopedic Surgery • Vol. 12, No. 3, $2020 \bullet$ www.ecios.org

ally develop cancer recurrence, of whom over $90 \%$ would succumb to this cancer. ${ }^{15)}$ Thus, more effective treatments are needed to improve outcomes in soft-tissue sarcoma. The CSC concept underlies a new, more effective treatment strategy - targeting a small subset of tumor-initiating populations with distinct properties. ${ }^{3)}$ The existence of CSCs in sarcomas has been reported using different identification methods in several subtypes of sarcomas. ${ }^{16}$ Characteristics of CSCs, such as the self-renewal ability and high tumorigenic potential, cause drug resistance and induce cancer relapses. ${ }^{11)}$ Therefore, the regulation of CSCs is important in the development of therapeutics for sarcoma.

DLK, a member of the notch/delta/serrate family, is preferentially expressed in immature cells with regenerative potential. DLK has been implicated in the Notch signaling pathway; it inhibits adipogenesis and seems to regulate the differentiation of hematopoietic stem cells. ${ }^{4)}$ Elevated expression of DLK1 is found in a variety of tumor cells including hepatoma and gliomas. ${ }^{10,11)}$ Other reports suggest that DLK1 may be associated with tumor cell differentiation and increased cell proliferation. ${ }^{10,17)}$ Nevertheless, the role of DLK1 in tumor progression remains poorly understood.

In this study, we isolated CSCs from sarcoma and investigated the role of DLK1 in sarcomas and sarcoma CSCs. The expression of DLK1 mRNA was higher in sarcoma cell lines than in normal bone cell lines, but there was no noticeable difference in DLK1 expression between different types of sarcomas. Surprisingly, the expression level of DLK1 protein was not proportional to the DLK1 mRNA expression level in some sarcomas. Previous reports have shown that, despite expressing high levels of DLK1 mRNA, sw872 cells express low levels of DLK1 protein. ${ }^{8)}$ Our results confirmed this expression pattern in sw872 as well as in A204 cells. We hypothesize that DLK1 may use alternative routes to protein expression to relay its signals. This may need to be investigated in other sarcoma types.

Several papers have already reported that DLK1 inhibits adipogenesis and proliferation in a variety of cells. ${ }^{17-20)}$ Our study also confirmed that suppressing DLK1 mRNA expression in sw872 did not decrease cell proliferation but rather increased the number of sw872 CSLCs. However, DLK1 suppression decreased cell proliferation in A204 and U2OS cells (Fig. 2). Thus, we think that DLK1 is involved in regulating cell proliferation. The FACS sorting method was used to sort the different sarcoma types, and the CD133 cell surface marker was used for CSLCs. The isolated CSCs displayed CSC characteristics and these were collected in a short time with a higher acquisition rate than the sphere-forming assay. When conducted using sphere-forming assays with FACS sorting, the isolation of CSCs was more efficient.

Soft agar assay was used to confirm the tumorigenic potential of the CSCs. Colonization of CSCs was activated in CD133- and wild-type cells except in A204 cells. In A204 cells, colonization was detected in all cell types. This suggests that in some cancer cells it is not possible to investigate the difference in tumorigenic potential between CSCs and non-CSLCs using soft agar assay.

The inhibition of DLK1 significantly decreased cell invasiveness in A204, U2OS, sw872, and CSCs of sw872. This reduction was more effective in the cell invasion than in the cell proliferation assay. Therefore, we think that DLK1 is involved in the regulation of tumorigenesis in sarcoma. In the study, we confirmed that the DLK1 gene is involved in regulating cell invasion in a variety of sarcomas. These results suggest that DLK1 may play an important role in the tumorigenesis and metastasis of sarcoma.

The novel finding is that we found the role of DLK1 in sarcoma. The proliferation of CSLCs (CD133+ cell or spheres) was higher than wild type similar to other articles. ${ }^{5-7)}$ But the expression of DLK1 in various sarcoma cell lines was decreased in CD133+ and spheres compared to wild-type or CD133- cells. Furthermore, when we performed knockdown DLK1 in sw872 cells, the proliferation and invasion ability of CD133- cells was much more suppressed than that of CD133+ cells or spheres, which may indicate that DLK1 has a greater role in proliferation of CD133 - cells than CSLCs.

However, more research is required to determine the exact signal mechanism of DLK1 and discover new factors that can aid the complete removal of sarcoma cells. We hope that our study will be beneficial to future researchers. In addition, our investigation of the relationship between the clinical prognosis and DLK1 expression in sarcoma highlights the clinical utility of DLK1.

\section{CONFLICT OF INTEREST}

No potential conflict of interest relevant to this article was reported.

\section{ACKNOWLEDGEMENTS}

The study was supported by the Seoul National University Research Fund (No. 0520120020). 
Kim et al. Delta-like Factor 1 as a Possible Therapeutic Target

Clinics in Orthopedic Surgery • Vol. 12, No. 3, $2020 \bullet$ www.ecios.org

\section{REFERENCES}

1. Bray F, Ferlay J, Soerjomataram I, Siegel RL, Torre LA, Jemal A. Global cancer statistics 2018: GLOBOCAN estimates of incidence and mortality worldwide for 36 cancers in 185 countries. CA Cancer J Clin. 2018;68(6):394-424.

2. Italiano A, Mathoulin-Pelissier S, Cesne AL, et al. Trends in survival for patients with metastatic soft-tissue sarcoma. Cancer. 2011;117(5):1049-54.

3. Clevers H. The cancer stem cell: premises, promises and challenges. Nat Med. 2011;17(3):313-9.

4. Ding XY, Ding J, Wu K, et al. Cross-talk between endothelial cells and tumor via delta-like ligand 4/Notch/PTEN signaling inhibits lung cancer growth. Oncogene. 2012;31(23):2899906.

5. Matushansky I, Charytonowicz E, Mills J, Siddiqi S, Hricik T, Cordon-Cardo C. MFH classification: differentiating undifferentiated pleomorphic sarcoma in the 21st century. Expert Rev Anticancer Ther. 2009;9(8):1135-44.

6. Naka N, Takenaka S, Araki N, et al. Synovial sarcoma is a stem cell malignancy. Stem Cells. 2010;28(7):1119-31.

7. Tirino V, Desiderio V, Paino F, et al. Human primary bone sarcomas contain CD133+ cancer stem cells displaying high tumorigenicity in vivo. FASEB J. 2011;25(6):2022-30.

8. Cheng H, Dodge J, Mehl E, et al. Validation of immature adipogenic status and identification of prognostic biomarkers in myxoid liposarcoma using tissue microarrays. Hum Pathol. 2009;40(9):1244-51.

9. Jing K, Heo JY, Song KS, et al. Expression regulation and function of Pref-1 during adipogenesis of human mesenchymal stem cells (MSCs). Biochim Biophys Acta. 2009;1791(8):816-26.

10. Jin ZH, Yang RJ, Dong B, Xing BC. Progenitor gene DLK1 might be an independent prognostic factor of liver cancer. Expert Opin Biol Ther. 2008;8(4):371-7.

11. Kim Y, Lin Q, Zelterman D, Yun Z. Hypoxia-regulated delta-like 1 homologue enhances cancer cell stemness and tumorigenicity. Cancer Res. 2009;69(24):9271-80.

12. Mohseny AB, Hogendoorn PC. Concise review: mesenchymal tumors: when stem cells go mad. Stem Cells. 2011;29(3): 397-403.

13. Taipaleenmaki H, Harkness L, Chen L, et al. The crosstalk between transforming growth factor- $\beta 1$ and delta like- 1 mediates early chondrogenesis during embryonic endochondral ossification. Stem Cells. 2012;30(2):304-13.

14. Patel NS, Dobbie MS, Rochester M, et al. Up-regulation of endothelial delta-like 4 expression correlates with vessel maturation in bladder cancer. Clin Cancer Res. 2006;12(16):4836-44.

15. Penel N, van Glabbeke M, Marreaud S, Ouali M, Blay JY, Hohenberger P. Testing new regimens in patients with advanced soft tissue sarcoma: analysis of publications from the last 10 years. Ann Oncol. 2011;22(6):1266-72.

16. Wu C, Wei Q, Utomo V, et al. Side population cells isolated from mesenchymal neoplasms have tumor initiating potential. Cancer Res. 2007;67(17):8216-22.

17. Abdallah BM, Jensen CH, Gutierrez G, Leslie RG, Jensen TG, Kassem M. Regulation of human skeletal stem cells differentiation by Dlk1/Pref-1. J Bone Miner Res. 2004;19(5):841-52.

18. Mortensen SB, Jensen CH, Schneider M, et al. Membranetethered delta-like 1 homolog (DLK1) restricts adipose tissue size by inhibiting preadipocyte proliferation. Diabetes. 2012;61(11):2814-22.

19. Rodriguez P, Higueras MA, Gonzalez-Rajal A, et al. The non-canonical NOTCH ligand DLK1 exhibits a novel vascular role as a strong inhibitor of angiogenesis. Cardiovasc Res. 2012;93(2):232-41.

20. Smas CM, Sul HS. Pref-1, a protein containing EGF-like repeats, inhibits adipocyte differentiation. Cell. 1993;73(4):72534. 